\title{
THREE-DIMENSIONAL MAGNETOGRADIENT WAVES IN THE UPPER ATMOSPHERE
}

\author{
${ }^{1}$ George Jandieri, ${ }^{2}$ Anzor Gvelesiani, ${ }^{3}$ Zhuzhuna Diasamidze, ${ }^{4}$ Mzia Diasamidze, \\ ${ }^{4}$ Irma Takidze \\ 1. Georgian Technical University, 77 Kostava Str., Tbilisi 0175, Georgia \\ E-mail: jandierigeorge7@gmail.com
}

2. M. Nodia Institute of Geophysics, 1 Aleksidze Str., Tbilisi 0193, Georgia

3. Batumi Shota Rustaveli State University, 35 Ninoshvili Str., 6010 Batumi, Georgia

E-mail: zhuzhuna.diasamidze@gmail.com

4. Batumi State Maritime Academy, 53 Rustaveli Av. 6010 Batumi, Georgia

E-mail: mzia.diasamidze@ gmail.com

\begin{abstract}
General dispersion equation has been obtained for three-dimensional electromagnetic planetary waves, from which follows, as particular case Khantadze results in one-dimension case. It was shown that partial magnetic field line freezingin as in one-dimension case lead to the excitation of both "fast" and "slow" planetary waves, in two-liquid approximation (i.e. at ion drag by neutral particles) they are represent oscillations of magnetized electrons and partially magnetized ions in $\mathrm{E}$ region of the ionosphere. In F region of the ionosphere using one-liquid approximation only "fast" planetary wave will be generated representing oscillation of medium as a whole. Hence, it was shown that three-dimension magnetogradient planetary waves are exist in all components of the ionosphere, and as exact solutions, with well-known slow short-wave MHD waves, are simple mathematical consequence of the MHD equations for the ionosphere.
\end{abstract}

\section{Indexing terms/Keywords}

Electromagnetic planetary waves, irregularities, ionosphere, MHD waves.

\section{Academic Discipline And Sub-Disciplines}

Physics, Plasma physics, Radio Physics.

\section{SUBJECT CLASSIFICATION}

Electromagnetic theory, Radio waves (theory), Plasma physics

\section{TYPE (METHOD/APPROACH)}

Complex geometrical optics approximation

\section{INTRODUCTION}

For the first time generalization of slow Rossby-type planetary waves taking into account latitudinal gradient of the geomagnetic field in the early seventies was given by Tolstoy [1] and independently by Khantadze [2]. Emphasizing their hydrodynamic nature, these waves Tolstoy called slow hydromagnetic gradient waves. In the subsequent papers [3,5-7] Khantadze for the first time shown that fast planetary waves of the electromagnetic nature should be exist in the Earth upper atmosphere, in both $\mathrm{E}$ and $\mathrm{F}$ regions of the ionosphere. These waves in $[8,9]$ were called magnetogradient waves of Khantadze. In the above-stated papers [2-7] classification of magnetogradient planetary waves (fast and slow), hydrodynamic end electromagnetic nature of these waves and the anisotropic nature of their propagation caused by curvature of lines of force of the geomagnetic field along the Earth parallels was for the first time given. Assessment of parameters of the considered waves, and also linear and nonlinear theories of magnetogradient waves, is given in [10-14]. Experimentally these waves were recorded in $[8,15,16]$. In the listed above papers generally one-dimensional and twodimensional magnetogradient waves propagation were considered. Meanwhile numerous observations confirm that the speed of propagation of large-scale wave perturbations having the electromagnetic nature except horizontal, always has vertical component, i.e. these waves are significantly three-dimensional $[8,15,16]$.

As is well-known [17-20], without compressibility and temperature stratification (excepting a planetary boundary layer of the troposphere) Coriolis force $\mathbf{F}_{C}=\rho[\mathbf{V} \cdot 2 \mathbf{\Omega}]$ becomes the main defining force in the equations of movement of the free atmosphere. The gyroscopic Coriolis force gives to the atmosphere additional stratification. In particular, angular velocity of the Earth rotation $\Omega$, which is function of latitude of the place $\varphi$, ingenerates the atmospheres speed gradients, and its latitudinal gradient $\nabla \Omega$ - inhomogeneity in the medium. 
As a result in internal waves in short-wave approximation $\left(\lambda \leq 10^{3} \mathrm{~km}\right)$, for which latitudinal variations $\Omega(\varphi)$ it is possible to neglect, inertial waves are exited in the atmosphere; and in long-wave approximation $\left(\lambda \sim 10^{3}-10^{4} \mathrm{~km}\right)$ when it is impossible to neglect latitudinal gradient of angular speed of Earth rotation $\nabla \Omega(\varphi)$, planetary Rossby waves are generated. For planetary-scale waves instead of Euler's equation it is necessary to use Helmholtz's equation for the velocity vortex naturally containing both size $\Omega$ and gradient of angular speed of rotation of Earth $\nabla \Omega$.

Indeed, in the considered approximation the three-dimension Helmholtz equation for a velocity vortex $\operatorname{rot} \mathbf{V}$ has the following form [17-19]:

$$
\frac{\partial \operatorname{rot} \mathbf{V}}{\partial t}+\operatorname{rot}[\operatorname{rot} \mathbf{V} \cdot \mathbf{V}]=(2 \mathbf{\Omega} \cdot \nabla) \mathbf{V}-(\mathbf{V} \cdot \nabla) 2 \mathbf{\Omega}
$$

from which visually follows that the first term in the right sight of equation (1) really generates in the atmosphere shortwave inertial waves [19], the second - Rossby planetary waves [17,18]. In absence of rotation of Earth wave movements disappear, and nonlinear Helmholtz equation for function of current will describe only convective movement of the atmosphere.

\section{MAGNETOHYDRODYNAMICS EQUATIONS OF THE IONOSPHERE OF INCOMPRESSIBLE ELECTROCONDUCTIVE LIQUID AND FORMULATION OF THE PROBLEM}

In this paper magnetogradient waves of Khantadze are generalized on three-dimensional case. In the upper atmosphere, since height of $130 \mathrm{~km}$ and above, the magnetic pressure of the geomagnetic field prevails over pressure of neutrals and ionospheric plasma. Therefore in the upper atmosphere in the wave processes proceeding in an ionosphere along with parameters $\Omega(\varphi)$ and $\nabla \Omega(\varphi)$, an essential role has be played the size of the geomagnetic field $\mathrm{H}_{0}\left(\varphi^{\prime}, r\right)$ and its gradients $\nabla \mathrm{H}_{0}\left(\varphi^{\prime}, r\right)$, where $r$-distance from the center of a geomagnetic dipole of Earth to the observation point, $\varphi^{\prime}$ is the geomagnetic latitude. As result from the general equations of magnetic hydrodynamics of the ionosphere neglecting compressibility and temperature stratification of the atmosphere, it is possible to receive the closed system of equations for $\mathbf{V}$ and $\mathbf{H}$ generalizing Helmholtz equation (1) and the equation of induction taking into account Hall's effect $[1,20]$ :

$$
\begin{gathered}
\frac{\partial \operatorname{rot} \mathbf{V}}{\partial t}+\operatorname{rot}[\operatorname{rot} \mathbf{V} \cdot \mathbf{V}]=\operatorname{rot}[\mathbf{V} \cdot 2 \mathbf{\Omega}]+\operatorname{rot} \frac{1}{4 \pi \rho}[\operatorname{rot} \mathbf{H} \cdot \mathbf{H}], \\
\frac{\partial \mathbf{H}}{\partial t}=\operatorname{rot}[\mathbf{V} \cdot \mathbf{H}]-\alpha \rho \operatorname{rot} \frac{1}{4 \pi \rho}[\operatorname{rot} \mathbf{H} \cdot \mathbf{H}], \\
\operatorname{div} \mathbf{V}=0, \operatorname{div} \mathbf{H}=0,
\end{gathered}
$$

where $\mathbf{V}=\mathbf{V}_{\mathbf{0}}+\mathbf{v}$ and $\rho$ are speed of ionospheric wind $\mathbf{V}_{\mathbf{0}}, \mathbf{v}$ - perturbation of velocity and medium density, $\mathbf{H}=\mathbf{H}_{\mathbf{0}}+\mathbf{h}, \mathbf{H}_{\mathbf{0}}$ - vector of the geomagnetic field, $\mathbf{h}$ - the perturbation of magnetic field caused by the medium motion, $\alpha=c / e N$ - Hall's parameter, $e$ - electron charge, $N$ - electron concentration, $c$ - speed of light, $\mathbf{F}_{\mathbf{A}}=[\operatorname{rot} \mathbf{H} \cdot \mathbf{H}] / 4 \pi \rho-$ electromagnetic Ampere force. Let's show that the considered closed system of equations of magnetic hydrodynamics of the ionosphere naturally contains the new exact solution as the three-dimensional magnetogradient planetary waves caused by the geomagnetic field of Earth $\mathbf{H}_{\mathbf{0}}$.

Taking into account that for the planetary-scale waves effects of compressibility and temperature stratification play a minor role $[6,17,18]$, we will seek the solution of this set of equations in the form of three-dimensional internal waves for a half-space [20]:

$$
\left.\mathbf{v}, \quad \mathbf{h} \sim A \exp \left(-\frac{\beta+g}{2 c_{S}^{2}} z_{*}\right) \exp \left[i\left(k_{x} x+k_{y} y+k_{z} z_{*}-\omega t\right)\right]=A \exp \left(-\frac{\beta+g}{2 c_{S}^{2}} z_{*}\right) \exp [i(\mathbf{k} \cdot \mathbf{r})-i \omega t)\right],
$$

where $k=2 \pi / \lambda$ - arbitrary wave number, $\omega$ - the frequency which should be definition, $z_{*}=z-z_{0}, z_{0}=80 \mathrm{~km}$, $\beta=(\kappa-1) g+\kappa R d \bar{T} / d z, c_{S}$ - sound speed, $\kappa$ - polytrope index, $R$ - gas constant, $g$ - acceleration of gravity, $\bar{T}(z)$ - 
temperature profile in the main state. In the incompressibility approximation $\left(c_{S} \rightarrow \infty\right)$ and arbitrary temperature stratification $(d \bar{T} / d z=0)$ amplitude of an internal wave becomes a constant and need of initial and boundary conditions disappears. Then the equations for the velocity vortex and induction in standard coordinate system ( $d x=r \sin \theta d \lambda$, $-d y=r d \theta, d r=d z, \quad x$ - axis is directed form the West to the East, $y$ - axis from the South on the North, $z$ - axis vertically up, $\theta=90^{0}-\varphi, \lambda$ - longitude) $[17,18]$ can be written in the form:

$$
\begin{gathered}
\frac{\partial \operatorname{rot} \mathbf{v}}{\partial t}+\operatorname{rot}[\operatorname{rot} \mathbf{v} \cdot \mathbf{v}]=\operatorname{rot}[\mathbf{v} \cdot 2 \mathbf{\Omega}]+\operatorname{rot}\left[\mathbf{u} \cdot 2 \mathbf{\Omega}_{\mathbf{H}}\right] \\
\frac{\partial \operatorname{rot} \mathbf{u}}{\partial t}+\operatorname{rot}[\operatorname{rot} \mathbf{u} \cdot \mathbf{u}]=\operatorname{rot}\left[\mathbf{v} \cdot 2 \mathbf{\Omega}_{\mathbf{i}}\right]-\delta \operatorname{rot}\left[\mathbf{u} \cdot 2 \mathbf{\Omega}_{\mathbf{H}}\right] \\
\operatorname{div} \mathbf{v}=0, \operatorname{div} \mathbf{u}=0 .
\end{gathered}
$$

where $\mathbf{u}$ is the vector potential of the vorticity determined by equation $\operatorname{rot} \mathbf{u}=\eta e \mathbf{h} / M c, \eta=N / N_{n}$ - iconicity of the ionosphere, $N$ and $N_{n}$ - concentration of plasma and neutrals, respectively, $M$ - mass of an ion. Vectors of angular speed of rotation of Earth $\boldsymbol{\Omega}$ and geomagnetic field $\mathbf{H}_{\mathbf{0}}$ in standard coordinate system have components: $\Omega_{x}=0$, $\Omega_{y}=\Omega_{0} \sin \theta, \Omega_{z}=\Omega_{0} \cos \theta, H_{0 x}=0, H_{0 y}=-H_{E} \sin \theta^{\prime}, H_{0 z}=-2 H_{E} \cos \theta^{\prime}, \theta^{\prime}=90^{0}-\varphi^{\prime}$ - magnetic co-latitude, $\Omega_{0}$ - module of the angular speed of rotation of Earth, $H_{E}$ - value of the geomagnetic field on the magnetic equator (further is accepted that geographical $\varphi, \lambda, r$ and geomagnetic $\varphi^{\prime}, \lambda^{\prime}, r^{\prime}$ coordinates coincide, i.e. is accepted that the geomagnetic dipole coincides with an axis of rotation of Earth). In the equation (3) $2 \mathbf{\Omega}_{\mathbf{i}}=\eta e \mathbf{H}_{\mathbf{0}} / M c-$ the modified cyclotron frequency of ions, $2 \mathbf{\Omega}_{\mathbf{H}}=c k^{2} \mathbf{H}_{\mathbf{0}} / 4 \pi e N=\left(c / c_{e}\right)^{2} e \mathbf{H}_{\mathbf{0}} / m c$ - the modified cyclotron frequency of electrons, $c_{e}=\omega_{e p} / k, \omega_{e p}=\left(4 \pi e^{2} N / m\right)^{1 / 2}$ - plasma frequency of electrons, $m$ - mass of an electron. As the charge of ions is positive and $H_{0}$ negatively, $2 \Omega_{\mathrm{i}}=\eta e \mathrm{H}_{0} / M c<0$, the charge of electrons is negative, $\omega_{e}=\left(e H_{0} / m c\right)>0$.

The solution of the considered set of equations is looked for in the form of three-dimensional internal waves $\exp [i(\mathbf{k} \cdot \mathbf{r})-i \omega t]$. Then expression of Ampere force $\mathbf{F}_{\mathbf{A}}=[\operatorname{rot} \mathbf{H} \cdot \mathbf{H}] / 4 \pi \rho$ in linear approximation equals $\mathbf{F}_{\mathbf{A}}=[\operatorname{rot} \mathbf{h} \cdot \mathbf{H}] / 4 \pi M N_{n}$. Entering vector potential $\mathbf{u}$ as $\operatorname{rot} \mathbf{u}=\eta e \mathbf{h} / M c$, from here $\mathbf{h}=M c \operatorname{rot} \mathbf{u} / \eta e$, and taking a rotor from $\mathbf{h}$, we will receive $\operatorname{rot} \mathbf{h}=M c \operatorname{rot} \operatorname{rot} \mathbf{u} / \eta e=-M c \Delta \mathbf{u} / \eta e$. Substituting magnetic field perturbation in this expression, we will obtain $\operatorname{rot} \mathbf{h}=M c k^{2} \mathbf{u} / \eta e$. Then Ampere's force $\mathbf{F}_{\mathbf{A}}$ we have

$$
\mathbf{F}_{\mathbf{A}}=\frac{1}{4 \pi M N_{n}} \frac{M c}{\left(N / N_{n}\right) e} k^{2}\left[\mathbf{u} \cdot \mathbf{H}_{\mathbf{0}}\right]=\left[\mathbf{u} \cdot \frac{c k^{2}}{4 \pi e N} \mathbf{H}_{\mathbf{0}}\right]=\left[\mathbf{u} \cdot 2 \mathbf{\Omega}_{\mathbf{H}}\right],
$$

i.e. $2 \boldsymbol{\Omega}_{\mathbf{H}}=c k^{2} \mathbf{H}_{\mathbf{0}} / 4 \pi e N$.

As vector $\mathbf{u}$ has dimension of speed, $2 \boldsymbol{\Omega}_{\mathbf{i}}$ and $2 \boldsymbol{\Omega}_{\mathbf{H}}$ have dimension of frequency $\left(\mathrm{s}^{-1}\right)$, this set of equations formally describes interaction of two incompressible liquids under the influence of three gyroscopic forces: Coriolis $\mathbf{F}_{\mathbf{C}}=\rho[\mathbf{V} \cdot 2 \boldsymbol{\Omega}]$ and electromagnetic gyroscopic forces, $\mathbf{F}_{\mathbf{i}}=\rho\left[\mathbf{V} \cdot 2 \mathbf{\Omega}_{\mathbf{i}}\right]$ and $\mathbf{F}_{\mathbf{H}}=\rho\left[\mathbf{u} \cdot 2 \mathbf{\Omega}_{\mathbf{H}}\right]$. The force $\mathbf{F}_{\mathbf{i}}$ is caused by vorticity of electric field, and $\mathbf{F}_{\mathbf{H}}$ - modified form of Ampere force $\mathbf{F}_{\mathbf{A}}$. Dimensionless parameter $\delta$ is entered for convenience: in $\mathrm{E}$ region of the ionosphere where Hall's effect plays an essential role, it is equal to unit $(\delta=1)$ and it is necessary to use three-liquid approximation for the ionosphere; and in $\mathrm{F}$ region of the ionosphere in which Hall effect is absent, parameter $\delta$ tends to zero and the ionosphere should be considered as single-fluid medium.

Thus, as well as in the case of the Coriolis gyroscopic force $\mathbf{F}_{\mathbf{C}}$, under the influence of the geomagnetic field the ionosphere gets additional stratification of the electromagnetic nature, and new wave branches of large-scale very lowfrequency (VLF) of electromagnetic waves which are caused by presence of gyroscopic electromagnetic forces are 
excited in the top atmosphere $\mathbf{F}_{\mathbf{i}}$ and $\mathbf{F}_{\mathbf{H}}$. In $\mathbf{E}$ region of the ionosphere force $\mathbf{F}_{\mathbf{i}}$ is comparable with the Coriolis Force $\mathbf{F}_{\mathbf{C}}$. Other gyroscopic force $\mathbf{F}_{\mathbf{H}}$ both in $\mathrm{E}$ and $\mathrm{F}$ regions of the ionosphere exceeds $\mathbf{F}_{\mathbf{C}}[7,11]$.

Linearizing the above-stated set of equations (2)-(4) in long-wave approximation $\left(\lambda \sim 10^{3}-10^{4} \mathrm{~km}\right)$ we will receive system of the equations which are investigated further $[7,11,20]$ :

$$
\begin{gathered}
\frac{\partial \operatorname{rot} \mathbf{v}}{\partial t}=-(\mathbf{v} \cdot \nabla) 2 \mathbf{\Omega}-(\mathbf{u} \cdot \nabla) 2 \mathbf{\Omega}_{\mathbf{H}}, \\
\frac{\partial \operatorname{rot} \mathbf{u}}{\partial t}=-(\mathbf{v} \cdot \nabla) 2 \mathbf{\Omega}_{\mathbf{i}}-\delta(\mathbf{u} \cdot \nabla) 2 \mathbf{\Omega}_{\mathbf{H}}, \\
\operatorname{div} \mathbf{v}=0, \quad \operatorname{div} \mathbf{u}=0 .
\end{gathered}
$$

Vector of the geomagnetic field $\mathbf{H}_{\mathbf{0}}$ satisfies Maxwell's equations: $\operatorname{rot} \mathbf{H}_{\mathbf{0}}=0$ and $\operatorname{div} \mathbf{H}_{\mathbf{0}}=0$. From here it is possible to enter the following two latitudinal gradients $\beta_{1}=\partial H_{0 z} / \partial y=\partial H_{0 y} / \partial z$ and $\beta_{2}=\partial H_{0 y} / \partial y=-\partial H_{0 z} / \partial z$. Further, as well as in the theory of long waves $\left(\lambda \sim 10^{3}-10^{4} \mathrm{~km}\right)$ Rossby, $\beta_{1}$ and $\beta_{2}$ are considered as constant and the set of equations (2)-(4) becomes equations with constant coefficients.

\section{DISPERSIAN EQUATIONS AND ESTIMATION OF THE WAVE PARAMETERS FOR THE SET OF EQUATIONS (5)-(7)}

Considering that the solution of system (5)-(7) as it was noted above, we look for in the form of internal three-dimensional plane waves $\mathbf{v}, \mathbf{u} \sim \exp \left[-i \omega t+i\left(k_{x} x+k_{y} y+k_{z} z\right)\right]$, neglecting for simplicity action of the Coriolis force, from (5)-(7) we will receive:

$$
\omega[\mathbf{k} \cdot \mathbf{v}]=-(\mathbf{u} \cdot \nabla) 2 \mathbf{\Omega}_{\mathbf{H}}, \omega[\mathbf{k} \cdot \mathbf{u}]=-(\mathbf{v} \cdot \nabla) 2 \mathbf{\Omega}_{\mathbf{i}}-\delta(\mathbf{u} \cdot \nabla) 2 \mathbf{\Omega}_{\mathbf{H}},(\mathbf{k} \cdot \mathbf{v})=0,(\mathbf{k} \cdot \mathbf{u})=0,
$$

where $(\mathbf{u} \cdot \nabla)=u_{y} \partial / \partial y+u_{z} \partial / \partial z,(\mathbf{v} \cdot \nabla)=v_{y} \partial / \partial y+v_{z} \partial / \partial z$.

Obvious equalities follow from the equations (8):

$$
\mathrm{v}_{y}=\frac{k_{y}}{k_{z}} \mathrm{v}_{z}, \quad \mathrm{v}_{x}=-\frac{k_{y}^{2}+k_{z}^{2}}{k_{x} k_{y}} \mathrm{v}_{y}, \quad \mathrm{v}_{x}=-\frac{k_{y}^{2}+k_{z}^{2}}{k_{x} k_{z}} \mathrm{v}_{z} ;
$$

similar expressions we yield for vector $\mathbf{u}$. Taking into account (9) the dispersion equation for three-dimensional waves of Khantadze can be easily obtain from the equation (8):

$$
\frac{\omega}{\omega_{H}}+\frac{\omega_{R_{0}}^{\prime}}{\omega}=\delta
$$

From here in $\mathrm{E}$ region of the ionosphere (where $\delta=1$ ), taking into account $\omega_{R}^{\prime}<<\omega[3,5]$ we will find the following two branches of oscillations:

a) for fast magnetogradient planetary wave (high-frequency branch):

$$
\omega \approx \omega_{H} \approx \omega_{H E} \frac{\sqrt{1+3 \sin ^{2} \theta}}{R_{0}} \frac{k_{x}}{k^{2}}=\frac{c H_{E}}{4 \pi e N} \frac{\sqrt{1+3 \sin ^{2} \theta}}{R_{0}} k_{x} ;
$$

where $\omega_{H E}=c k^{2} H_{E} / 4 \pi e N=\left(e / c_{e}\right)^{2} e H_{E} / m c$ is the eigenfrequency of the magnetized electrons (helicons), $R_{0}$ Earth's radius.

b) for a slow low-frequency Rossby-type planetary wave we have:

$$
\omega \approx \omega_{R o}^{\prime} \approx-\Omega_{i E} \frac{\sqrt{1+3 \sin ^{2} \theta}}{R_{0}} \frac{k_{x}}{k^{2}} .
$$


where $\Omega_{i E}=\eta e H_{E} / M c$ - eigenfrequency of VLF ion-cyclotron waves; $\omega_{H E}$ and $\Omega_{i E}$ - their values on the equator.

In $\mathrm{F}$ region of the ionosphere $(\delta=0)$ we will have only one branch of the fast magnetogradient planetary waves propagating both in positive and negative directions:

$$
\omega=\omega_{n}= \pm \omega_{A E} \frac{\sqrt{1+3 \sin ^{2} \theta}}{R_{0}} \frac{k_{x}}{k^{2}}
$$

where $\omega_{A E}=\sqrt{\eta} k H_{E} / \sqrt{4 \pi M N}=k V_{A E}$ - eigenfrequency of slow Alfven wave $\left(V_{A E}\right)$ on the equator. In expressions (11)-(13), except Earth radius $R_{0}$, which naturally determines the spatial scale and long-periodicity of electromagnetic planetary waves, only two parameters enter: $N$ and $\eta$. Concentration $N$ in the ionosphere varies with altitude only from $10^{4}-10^{5} \mathrm{~cm}^{-3}$, and the ionicity $\eta$ in the daytime conditions has the following values $[9,21]$ :

\begin{tabular}{|c|c|c|c|c|c|c|c|c|}
\hline$z, \mathrm{~km}$ & 100 & 120 & 150 & 200 & 250 & 300 & 350 & 400 \\
\hline$\eta$ & $10^{-8}$ & $10^{-7}$ & $10^{-6}$ & $10^{-5}$ & $10^{-4}$ & $10^{-3}$ & $10^{-3}$ & $10^{-2}$ \\
\hline
\end{tabular}

For night conditions at all altitudes $\eta$ decreases by an order of magnitude. Using these ionospheric parameters below we calculate eigenfrequency of considered waves. As vorticities of electrons $2 \boldsymbol{\Omega}_{\mathbf{H}}$ and ions $2 \boldsymbol{\Omega}_{\mathbf{i}}$ in the geomagnetic field $\mathbf{H}_{\mathbf{0}}$ are directed from the South to the North and from the North on the South (i.e. electrons rotate in the geomagnetic field anticlockwise direction, and ions in the clockwise direction), frequency (11), as well as in inertial waves [19], only positive value, and frequency (12) - negative has.

Frequency square $\omega_{n}^{2}=-\omega_{H} \cdot \omega_{R_{0}}^{\prime}$ does not depends on the vorticity and, as well as in Alfven waves, frequency (13) naturally gives two frequencies $\pm \omega_{n}$. Also follows from formula (12) that unlike three-dimensional Rossby wave $\omega_{R o}=-\beta k_{x} / k^{2}, \quad \beta=\partial \omega_{0 z} / \partial y=2 \Omega_{0} \sin \theta k_{x} / R_{0} k^{2} \quad$ [18], propagating mainly to the western direction, slow magnetogradient waves always have east phase speed. Obviously it is caused by the fact that vectors $\mathbf{\Omega}$ and $\mathbf{H}_{\mathbf{0}}$ are directed opposite to each other: $\boldsymbol{\Omega}$ - from the South on the North, $\mathbf{H}_{\mathbf{0}}-$ from the North on the South. At $k_{z}=0$ expressions (11)-(13) coincide with results [6]. In view of the fact that gyroscopic forces $\mathbf{F}_{\mathbf{i}}$ and $\mathbf{F}_{\mathbf{H}}$ do not make work, the kinetic energy concluded in magnetogradient waves remains completely.

From the expressions (11)-(13) follow that the phase speed of fast planetary waves (formula (11)) does not depend on the wave number, do not experience dispersion and they propagate one-dimensional, however slow Rossby type waves in $\mathrm{E}$ region and fast waves in $\mathrm{F}$ region of the ionosphere are strongly dispersive. The considered waves have all-planetary character and they can be exited at all latitudes of Earth. As in planetary waves horizontal wavenumbers satisfy the conditions $k_{x}, k_{y}<<k_{z}$, the formulas (11)-(13) received above can be simplified:

$$
\begin{gathered}
\omega=\omega_{H} \frac{c H_{E}}{4 \pi e N} \frac{\sqrt{1+3 \sin ^{2} \theta}}{R_{0}} k_{x}, \\
\omega \approx \omega_{R o}^{\prime}=-\eta \omega_{i E} \frac{\sqrt{1+3 \sin ^{2} \theta}}{R_{0}} \frac{k_{x}}{k_{z}^{2}}, \\
\omega=\omega_{n}= \pm V_{A E} \frac{\sqrt{1+3 \sin ^{2} \theta}}{R_{0}} \frac{k_{x}}{k_{z}} .
\end{gathered}
$$

Vertical group speed of waves $c_{g r, z}=\partial \omega / \partial k_{z}$ taking into account a sign $\omega_{R o}^{\prime}$ and $\omega_{n}$ define the direction of transfer of wave energy. In a case $c_{g r, z}>0$ energy of waves is transferred from the lower layers of the ionosphere in upper; and in a 


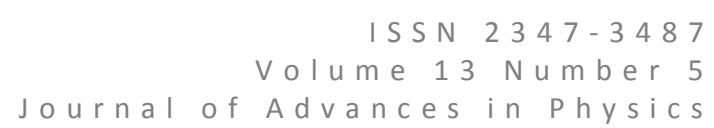

case $c_{g r, z}<0$ - on the contrary, from the upper layers of the ionosphere to the lower one. Now, at least, two permanently thermal sources of waves in the upper atmosphere are well-known: one of them is at the height of $80 \mathrm{~km}$, where because of strong turbulence of the environment there is an attenuation of the acoustic-gravity waves, tidal and planetary waves going from below, and the second - a high-latitude thermal source at the heights of 350-400 km, where kinetic energy of high energetic magnetosphere particles going from above turns into heat [20]. Characteristic vertical linear scale of planetary waves - an order of a scale of heights, which in the troposphere is about $8 \mathrm{~km}$, and $30 \mathrm{~km}$ in $\mathrm{E}$ region and $50 \mathrm{~km}$ in $\mathrm{F}$ region of the ionosphere. Characteristic horizontal linear scales of planetary waves along parallel and along meridian are an order of $10^{3}-10^{4} \mathrm{~km}$. Therefore the ionosphere for such large-scale wave processes is represented in the form of a thin film and analytical consideration of waves can be carried out according to the known theory of "small water" [17,18]. For planetary waves owing to these conditions $k_{x}, k_{y}<<k_{z}$ full wave vector $\mathbf{k}$ will have the direction, close to a vertical. Let's note that such inclination of the line of constant phase of planetary waves very often is registered at observation in the atmosphere [18].

It is also necessary to note that the considered fast magnetogradient waves, as shown in [9], transfer ionospheric perturbations on global distances along parallels and meridians. Numerical values of phase speed of fast magnetogradient $c_{H}$ - waves, calculated using experimental data are provided in [8] where it is shown that parameters $c_{H}$ - Khantadze's waves in $\mathrm{E}$ region of the ionosphere lie in the interval: $\lambda \sim\left(10^{3}-10^{4}\right) \mathrm{km}, \omega_{H} \sim\left(10^{-1}-10^{-4}\right) \mathrm{s}^{-1}$ and $c_{H} \sim(0.1-0.7)$ $\mathrm{km} \mathrm{s}^{-1}$ and $(0.5-7) \mathrm{km} \mathrm{s}^{-1}$ for day and night conditions, respectively. For magnetic field perturbations we have $\sim(2-100)$ $\mathrm{nT}$ (nanotesla), when ionospheric parameters change within 10-100\%. Calculation of parameters for a slow hydromagnetic wave $c_{R_{0}}$ carried out by us, have values: $\lambda \sim\left(10^{3}-10^{4}\right) \mathrm{km}, \omega_{R o}^{\prime} \sim\left(10^{-4}-10^{-6}\right) \mathrm{s}^{-1}$, and $c_{R o}^{\prime} \sim\left(10-10^{2}\right) \mathrm{m} \mathrm{s}^{-1}$. For magnetic field perturbation we have (1-20) nT when ionospheric parameters change within $30-80 \%$. In $\mathrm{F}$ region of the ionosphere for fast magnetogradient $c_{n}$-wave we received: $\lambda \sim\left(10^{3}-10^{4}\right) \mathrm{km}, \quad \omega_{n} \sim\left(3-10^{-3}\right) \mathrm{s}^{-1}$, $c_{n} \sim(10-50) \mathrm{km} \mathrm{s}^{-1}$. Magnetic perturbations change from several units to several hundred $\mathrm{nT}$ when ionospheric parameters change from 0,5 to $30 \%$. Numerical values of parameters $c_{n}$ - waves essentially depend on magnetic activity of the Sun. In view of that this paper has character of brief communication we refrain from further specification of the considered waves. In more detail numerical values of parameters of magnetogradient waves, their altitude profiles for different levels of activity of the Sun, time of day and seasons are carried out in [21].

\section{CONCLUSION}

Summarizing, we can claim that unlike two-dimensional planetary waves which can extend only in the horizontal direction three-dimensional slow and fast magnetogradient planetary waves should mainly propagate in the vertical direction $\left(k_{z}>k_{x}, k_{y}\right)$, which well is confirmed by observations in the upper atmosphere $[15,20]$.

\section{ACKNOWLEDGMENTS}

This work has been supported by the International Science and Technology Center (ISTC) under Grant \# G-2126 and Shota Rustaveli National Science Foundation under Grant \# FR/3/9-190/14.

\section{REFERENCES}

1. Tolstoy, I. 1967. Hydromagnetic gradient waves in the ionosphere, J. Geophys. Res. vol. 72, 1435-1442.

2. Khantadze, A.G. 1967. About definition of movement versus pressure and latitudinal effect of the geomagnetic field, Proceedings of the Geophysics Institute Georgian Academy of Sciences, 24-29 (in Russian).

3. Khantadze, A.G. 1986. Hydromagnetic gradient waves in the dynamo-region of the ionosphere, Bulletin of the Georgian Academy of Sciences, vol. 123, \# 1, 69-71, (in Russian).

4. Kobaladze Z.L., and Khantadze A.G. 1989. Propagation of large-scale perturbations in the ionosphere, Bulletin of the Georgian Academy of Sciences, vol. 134, 97-100 (in Russian).

5. Khantadze A.G. 1999. On the electromagnetic planetary waves in the Earth's ionosphere, J. Georgian Geophys. Soc. vol. 4B, 125-127 (in Russian).

6. Khantadze A.G. 2001. About new branch of the self-oscillation of the conducting atmosphere. Doklady Earth Science, vol. 376, \# 2, 250-252. 
7. Khantadze A.G. 2005. Electromagnetic planetary waves in the Earth atmosphere, Geomagnetizm and Aeronomy, vol. 42, \# 3, 333-335.

8. Burmaka V.P., Kostrov L.S., and Chernogor L.F. 2003. Statistics of signals of HF Doppler radar probing the bottomside ionosphere distributed by rocket launches and solar terminator, Radio Physics and Radio Astronomy, vol. 8, \# 2 143162, (in Russian).

9. Chernogor L.F. 2003. Earth science, atmosphere and geocosmos in the paradigm system point of view," Radio Physics and Radio Astronomy, vol. 8, \# 1, 59-106 (in Russian).

10. Khantadze A.G., Aburjania G.D., and Lominadze J.G. 2006. New branches of self-ULF electromagnetic oscillations of the ionospheric resonator," Doklady Earth Science, vol. 406, \# 2, 244-248.

11. Aburjania G.D., Chargazia K.E., Jandieri G.V., Khantadze A.G., and Kharshiladze O.A. 2004. On the new modes of planetary electromagnetic waves in the ionosphere," Annales Geophysicae, vol. 22, 1-9.

12. Aburjania G.D., Jandieri G.V., and Khantadze A.G. 2003. Self-organization of planetary-scale electromagnetic waves in the ionosphere, J. Atmos. Terr. Phys. vol. 65, 661-671.

13. Aburjania G.D., Chargazia K.E., Jandieri G.V., Khantadze A.G., and Lominadze J.G. 2005. Generation and propagation of the ULF planetary-scale electromagnetic wavy structures in the ionosphere," Planet. Space Sci. vol. 53, 881-901.

14. Aburjania G.D., and Khantadze A.G. 2006. Mechanism of the planetary Rossby wave energy amplification and transformation in the ionosphere with an inhomogeneous zonal smooth shear wind, J. Geophys. Res. vol. 111, 1-17, AO11567, 2006.

15. Sharadze Z.S. Atmospheric waves in the middle-latitude ionosphere. Doctor Dissertation Moscow, 255 p., 1991.

16. Fagundes P.R., Pillat V.G., Bolzan M. J. A., et al. 2005. Observations of F layer electron density profiles modulated by planetary wave type oscillations in the equatorial ionospheric anomaly region," J. Geophys. Res. vol. 110, pp. 1302.

17. Pedlosky J. Geophysicae Fluid Dynamics, Springer, Berlin, 1987.

18. Gill A., Atmosphere-Ocean Dynamics, Academic Press, New York, 1986.

19. Landau L.D., Lifshits E.M. Hydrodynamics. Nauka, Moscow, 1988 (Translated in English)

20. Khantadze A.G. Some Problems of the Dynamic of Conducted Atmosphere. Tbilisi, Mecniereba, 1973.

21. Khantadze A.G., Aburjania G.D., and Gvelesiani A.I. 2003. Physics of new branches of the planetary electromagnetic waves in the ionosphere, Geomagnetizm and Aeronomy. vol 43, 193-198. 